\title{
Evaluation de l'effet d'un extrait aqueux de Bridelia ferruginea Benth. (Euphorbiaceae) sur les activités spontanée et locomotrice chez le rat
}

\author{
Semi Anthelme NENE BI ${ }^{1^{*}}$, Ouga Stanislas ZAHOUI ${ }^{1}$, Tianga Yaya SORO ${ }^{1}$ \\ et Flavien TRAORE ${ }^{1}$ \\ ${ }^{1}$ Laboratoire de Physiologie Animale, Unité de Formation et de Recherche (UFR) Biosciences, Université de \\ Cocody-Abidjan, 22 BP 582 Abidjan 22, Côte d'Ivoire. \\ *Auteur correspondant, E-mail: neneanthelme@gmail.com,Tel : (225) 05068986
}

\section{RESUME}

L'objectif de ce travail est d'évaluer l'effet d'un extrait aqueux de l'écorce de tige de Bridelia ferruginea (SEA) sur le système nerveux central chez le rat. L'extrait aqueux de cette plante est administré aux animaux par la voie intrapéritonéale 30 minutes avant l'évaluation. L'actographe a permis de quantifier l'activité spontanée alors que l'activité locomotrice a été évaluée grâce à la roue d'activité et la traction. SEA, à la dose de $10 \mathrm{mg} / \mathrm{kg}$ de poids corporel réduit de manière significative l'activité spontanée de $63,26 \%(P<0,001)$ et de $81,52 \%(P<0,01)$ chez les rats pour les intervalles de temps respectifs de 5 et 10 minutes. L'activité locomotrice chez ces animaux est réduite de 78,08\% $(P<0,0001) 15$ minutes après l'enregistrement de cette activité. Cette dose augmente la latence du réflexe de rétablissement chez le rat. Ces résultats suggèrent que SEA possède un effet sédatif. Cet effet serait lié à la présence d'alcaloïde.

(C) 2010 International Formulae Group. All rights reserved.

Mots clés: Bridelia ferruginea, Effet sédatif, Alcaloïde, Antipsychotique.

\section{INTRODUCTION}

Bridelia ferruginea est une plante commune à toutes les savanes de la zone guinéenne au sahel et s'étendant de la Guinée en Angola (Dembélé, 1988; Adjanohoun et al., 1989).

En Côte d'Ivoire, elle est abondamment représentée depuis les savanes pré-lagunaires jusqu'au Nord (Adjanohoun et Ake-assi, 1979). On la trouve dans toute l'Afrique intertropicale (Ake-assi et Guinko, 1991). Dans la pharmacopée africaine, Bridelia ferruginea est utilisée dans le traitement de l'hypertension (Diafouka, 1997), de la varicelle, des maux de dents et de l'infection pulmonaire (Bitsindou et Lejoly, 1996 ; Ambe et Malaisse, 2000).

Selon Adjanohoun et al. (1989), l'écorce de tige de Bridelia ferruginea serait utilisée comme sédatif, tranquillisant, calmant et agirait comme un somnifère.

Des études pharmacologiques des extraits aqueux, éthanolique et méthanolique ont montré que cette plante aurait des propriétés anti-inflammatoire, antimicrobienne et anti-thrombique (Olajide et al., 1999 ; Olajide et al., 2000; Olajide et al., 2003). D'après Néné bi et al. (2008), l'extrait 
aqueux de l'écorce de tige de cette plante aurait un effet hypotenseur. L'étude phytochimique de l'extrait aqueux de cette plante a révélé la présence des quinones, des tanins, des alcaloïdes, des stérols et polyterpènes, polyphénols, des composés réducteurs, des saponines et des flavonoïdes (Néné bi et al., 2009).

Le but de ce travail est d'évaluer l'effet de l'extrait aqueux de Bridelia ferruginea et de comparer son action à ceux de deux médicaments psychotropes (chlorpromazine ${ }^{\circledR}$ et d'adrafinil ${ }^{\circledR}$ ) sur le système nerveux central chez le rat.

\section{MATERIEL ET METHODES}

Le matériel végétal utilisé est constitué d'écorces de tige de Bridelia ferruginea. Ces écorces ont été identifiées par un expert botaniste, le Professeur Aké-Assi Laurent du Centre National de floristique de Côte d'Ivoire (Université de Cocody-Abidjan) où se trouve conservé un échantillon de cette plante (herbier $n^{\circ} 17148$ du 19 Août 1985).

\section{Préparation de l'extrait aqueux de Bridelia ferruginea}

Les écorces ont été séchées à l'ombre à une température comprise entre $26{ }^{\circ} \mathrm{C}$ et 30 ${ }^{\circ} \mathrm{C}$ puis finement broyées à l'aide d'un microbroyeur Culatti (France). Le broyat obtenu $(50 \mathrm{~g})$ est mis en macération dans un litre d'eau distillée pendant 24 heures sous agitation magnétique. Le surnageant obtenu est filtré sur papier wattman $n^{\circ} 1$. Le filtrat est évaporé à $70{ }^{\circ} \mathrm{C}$ à l'aide d'un rotavapor de type BUCHI (France). La pâte obtenue est congelée à $-30{ }^{\circ} \mathrm{C}$ et lyophilisée à $-45{ }^{\circ} \mathrm{C}$, grâce à un lyophilisateur de type TELSTAR (Espagne). Le lyophilisat est réduit en poudre et conservé au réfrigérateur à $-5^{\circ} \mathrm{C}$.

\section{Animaux}

Les animaux utilisés pour l'expérimentation sont des rats mâles de souche Wistar pesant entre 150 et 200 g. Ils proviennent d'un élevage de l'UFR
Biosciences de l'Université de CocodyAbidjan. Les tests sont effectués dans une salle exempte de bruits, à la température ambiante $\left(25 \pm 2{ }^{\circ} \mathrm{C}\right)$. Les animaux utilisés pour les tests sont gardés en cage, dans la pièce d'expérimentation au moins 24 heures avant le test, pour les habituer à l'ambiance de la salle.

Les solutions à tester sont injectées par voie intrapéritonéale. Les expériences antérieures réalisées, nous ont permis de retenir, pour chaque substance, la dose à tester. Chaque rat sera successivement testé après injection du solvant $(\mathrm{NaCl} 9 \%$ ) et après injections des différentes drogues que sont la chlorpromazine (2,5 $\mathrm{mg} / \mathrm{kg}$ de poids corporel), l'adrafinil (10 mg/kg de poids corporel) et l'extrait aqueux de Bridelia ferruginea (10 $\mathrm{mg} / \mathrm{kg}$ de poids corporel). Au total, 6 animaux ont été utilisés pour chaque type de drogue. Les animaux ont été traités conformément aux principes du comité d'éthique concernant l'utilisation des animaux de laboratoire dans des tests d'expérimentation. La chlorpromazine $^{\circledR}$ provient du laboratoire Sanofi aventis (France) et l'adrafinil $^{\circledR}$ du laboratoire Cephalon (France).

\section{Appareils de mesure des activités du rat Actographe}

L'actographe est une cage en plexiglas dont le plateau est équipé de cellules photoélectriques qui captent chaque déplacement de l'animal. Durant 15 minutes, le compteur marque, par séquences de 5 minutes, le nombre de fois que l'animal traverse un faisceau photoélectrique. Ces déplacements sont le reflet de l'activité exploratoire de l'animal.

\section{Roue d'activité}

L'animal est introduit dans une cage en forme de roue. Chaque déplacement de l'animal imprime à cette roue un mouvement de rotation. Le nombre de rotations, chiffré par le compteur, durant 15 minutes, permet de quantifier l'activité motrice. 


\section{Traction}

Ce dispositif est constitué d'une barre de fer tendue entre deux potences.

L'animal tenu par la queue est approché à la barre de fer de telle façon qu'il s'y agrippe par les pattes avant et reste suspendu. Le chronomètre est alors mis en marche pour déterminer le temps que met le rat pour poser une patte arrière sur la barre de fer.

Cette manipulation permet de déterminer la latence du réflexe de rétablissement.

\section{Traitement des résultats expérimentaux}

L'analyse statistique des résultats a été réalisée grâce à l'analyse des variances (ANOVA) du test de comparaison multiple de Tukey-Kramer. $\quad P<0,05$ est considéré significatif. Toutes les valeurs sont présentées sous la forme moyenne \pm ESM.

\section{RESULTATS}

Effet de SEA sur l'activité motrice spontanée

Une seule dose $(10 \mathrm{mg} / \mathrm{kg})$ de notre extrait a été testée sur l'activité motrice spontanée chez le rat. Les résultats de la Figure 1 montre l'activité motrice spontanée mesurée pour les intervalles de temps de 5 ; 10 et 15 minutes. Après administration de SEA, l'activité motrice spontanée chez les rats est diminuée de $63,26 \%(P<0,001)$ et de $81,52 \% \quad(P<0,01)$ respectivement pour les intervalles de 5 et 10 minutes comparée au témoin. A ces mêmes intervalles de temps, la chlorpromazine $(2,5 \mathrm{mg} / \mathrm{kg})$ entraîne une diminution significative de cette activité respectivement de $89,99 \%(P<0,001)$ et de $96,33 \%(P<0,001)$ par rapport au témoin. L'adrafinil $(10 \mathrm{mg} / \mathrm{kg})$, pour ces mêmes intervalles de temps, provoque une augmentation de l'activité motrice spontanée de $80,08 \%(P<0,001)$ et de $143,18 \%(P<0,01)$ comparée au témoin. Aux intervalles de temps de 5 et 10 minutes, l'administration de SEA et de la chlorpromazine provoquent une réduction significative de l'activité spontanée contrairement à l'adrafinil qui entraîne l'augmentation de cette activité (Tableau 1).

Après 15 minutes, cette activité est réduite de manière significative $(P<0,001)$ chez les rats traités avec SEA et la chlorpromazine. Par contre, cette activité est augmentée chez les animaux ayant reçu l'adrafinil. Cette augmentation est statistiquement significative $\quad(P<0,001)$ comparée au témoin (Figure 1).

\section{Effet de SEA sur l'activité locomotrice}

Pour cette expérience, une seule dose de SEA $(10 \mathrm{mg} / \mathrm{kg})$ a été administrée à chaque rat du lot. L'activité locomotrice chez ces animaux a été mesurée sur des intervalles de 5,10 et 15 minutes, 30 minutes après l'administration des différentes substances. Pour les intervalles de temps de 5 et 10 minutes, l'activité locomotrice chez les animaux traités avec SEA est diminuée de manière significative comparée à la valeur témoin. La chlorpromazine $(2,5 \mathrm{mg} / \mathrm{kg})$, après son administration réduit de façon significative l'activité locomotrice chez les rats traités. L'adrafinil $(10 \mathrm{mg} / \mathrm{kg})$, quant à lui, provoque une augmentation de l'activité locomotrice (Tableau 2).

A la Figure 2, cette activité est réduite de $78,08 \%(P<0,001)$ et $85,15 \%(P<0,001)$, après 15 minutes d'enregistrement, chez les rats ayant reçu respectivement SEA et la chlorpromazine.

Par contre, cette même activité est augmentée de $75,30 \% \quad(P<0,001)$ chez les animaux traités avec l'adrafinil.

\section{Effet de SEA sur la latence du réflexe de rétablissement}

Après l'administration de SEA (10 $\mathrm{mg} / \mathrm{kg}$ ) aux rats, le temps de latence du réflexe de rétablissement qui était de $1,33 \pm 0,21$ secondes chez le groupe témoin, augmente et atteint 3,17 $\pm 0,4$ secondes. L'injection de SEA provoque donc une augmentation de $138,35 \%(P<0,0056)$ du temps de latence du 
Tableau 1: Etude comparée des effets de SEA, de la chlorpromazine et de l'adrafinil sur l'activité spontanée du rat en fonction du temps.

\begin{tabular}{|c|c|c|c|c|c|c|}
\hline \multirow[t]{2}{*}{$\begin{array}{l}\text { Temps } \\
(\text { min) }\end{array}$} & \multicolumn{2}{|c|}{$\begin{array}{c}\text { SEA } \\
(10 \mathrm{mg} / \mathrm{kg} \text { de PC) }\end{array}$} & \multicolumn{2}{|c|}{$\begin{array}{c}\text { Chlorpromazine } \\
(2,5 \mathrm{mg} / \mathrm{kg} \text { de PC) }\end{array}$} & \multicolumn{2}{|c|}{$\begin{array}{c}\text { Adrafinil } \\
(10 \mathrm{mg} / \mathrm{kg} \text { de PC) }\end{array}$} \\
\hline & Témoin & Test & Témoin & Test & Témoin & Test \\
\hline ]0 -5] & $\begin{array}{c}75,33 \pm \\
11,53\end{array}$ & $\begin{array}{c}27,67 \pm 1,36 \\
* * *\end{array}$ & $\begin{array}{c}92,17 \pm \\
5,11\end{array}$ & $\begin{array}{c}9,33 \pm 1,96 \\
* * *\end{array}$ & $89,5 \pm 9,37$ & $\begin{array}{c}161,17 \pm \\
13,10 * * *\end{array}$ \\
\hline ] 5 -10] & $39,67 \pm 5,24$ & $\begin{array}{c}7,33 \pm 2,25 \\
* *\end{array}$ & $37,5 \pm 5,25$ & $\begin{array}{c}2,67 \pm 1,31 \\
* * *\end{array}$ & $37,17 \pm 4,96$ & $79 \pm 5,94 * *$ \\
\hline ]10 - 15] & $8,83 \pm 0,87$ & $2,33 \pm 1,23$ & $9 \pm 2,63$ & $0,33 \pm 0,21$ & $8,5 \pm 1,12$ & $20,67 \pm 0,76$ \\
\hline
\end{tabular}

SEA et la chlorpromazine réduisent l'activité spontanée du rat contrairement à l'adrafinil.

Les valeurs expriment l'activité spontanée du rat en fonction de la substance administrée et en fonction du temps (Moyenne \pm $\mathrm{ESM}, * * \mathrm{p}<0,01, * * * \mathrm{p}<0,001, \mathrm{n}=6)$.

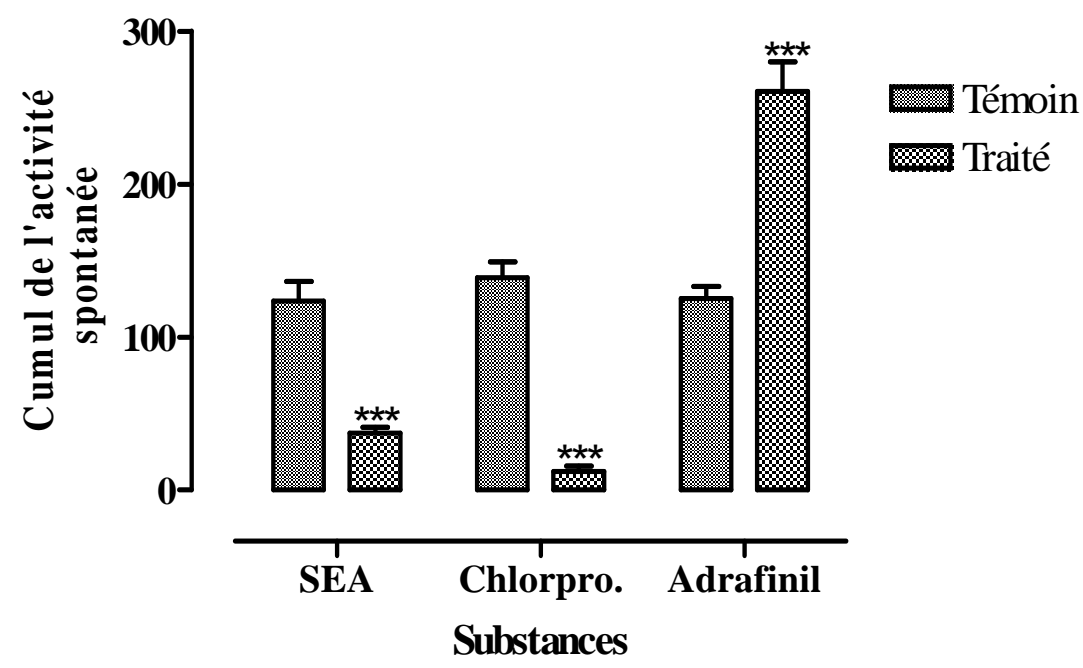

Figure 1: Cumul de l'activité spontanée du rat, 15 minutes après administration de SEA, de la chlorpromazine et de l'adrafinil. Chlorpro. = Chlorpromazine ; SEA = Extrait aqueux de l'écorce de $B$. ferruginea . SEA, comme la chlorpromazine, diminue l'activité spontanée du rat. SEA a un effet inverse de celui de l'adrafinil. Les valeurs expriment l'activité spontanée du rat enregistrée pendant 15 minutes après administration de SEA, de la chlorpromazine et de l'adrafinil (Moyenne \pm ESM, ***p<0,001, $\mathrm{n}=6$ ). 
Tableau 2: Etude comparée des effets de SEA, de la chlorpromazine et de l'adrafinil sur l'activité locomotrice du rat en fonction du temps.

\begin{tabular}{|c|c|c|c|c|c|c|}
\hline \multirow[t]{2}{*}{$\begin{array}{l}\text { Temps } \\
\text { (min) }\end{array}$} & \multicolumn{2}{|c|}{$\begin{array}{c}\text { SEA } \\
(10 \mathrm{mg} / \mathrm{kg} \text { de } \mathrm{PC})\end{array}$} & \multicolumn{2}{|c|}{$\begin{array}{c}\text { Chlorpromazine } \\
(2,5 \mathrm{mg} / \mathrm{kg} \text { de } \mathrm{PC})\end{array}$} & \multicolumn{2}{|c|}{$\begin{array}{c}\text { Adrafinil } \\
(10 \mathrm{mg} / \mathrm{kg} \text { de } \mathrm{PC})\end{array}$} \\
\hline & Témoin & Test & Témoin & Test & Témoin & Test \\
\hline$] 0-5]$ & $36,83 \pm 4,06$ & $\begin{array}{l}9,5 \pm 1,77 \\
* * *\end{array}$ & $\begin{array}{c}39,67 \pm \\
4,07\end{array}$ & $\begin{array}{c}7,5 \pm 0,76 \\
* * *\end{array}$ & $38,5 \pm 5,61$ & $\begin{array}{c}74,17 \pm 5,83 \\
* * *\end{array}$ \\
\hline$[5-10]$ & $9,17 \pm 1,42$ & $1 \pm 0,37 * *$ & $\begin{array}{c}12,67 \pm \\
2,01\end{array}$ & $\begin{array}{l}0,5 \pm 0,34 \\
\quad * *\end{array}$ & $14,83 \pm 2,07$ & $18,5 \pm 0,99$ \\
\hline ]10 - 15 ] & $2,67 \pm 1,31$ & $0,17 \pm 0,17$ & $\begin{array}{c}2,67 \pm \\
0,95\end{array}$ & $0,17 \pm 0,17$ & $4 \pm 1,39$ & $7,83 \pm 2,27$ \\
\hline
\end{tabular}

SEA et la chlorpromazine réduisent l'activité locomotrice du rat contrairement à l'adrafinil. Les valeurs expriment l'activité locomotrice du rat en fonction de la substance administrée et en fonction du temps (Moyenne \pm ESM, $* * p<0,01, * * * p<$ $0,001, \mathrm{n}=6)$.

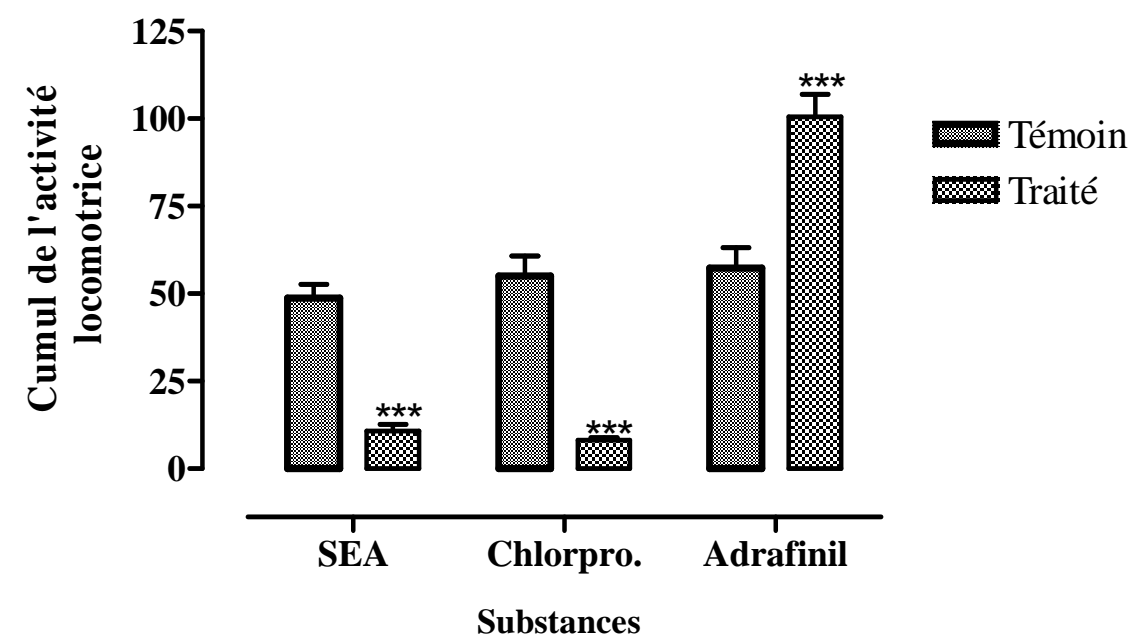

Figure 2: Cumul de l'activité locomotrice du rat, 15 minutes après administration de SEA, de la chlorpromazine et de l'adrafinil. Chlorpro. = Chlorpromazine $; \mathrm{SEA}=$ Extrait aqueux de l'écorce de $B$. ferruginea SEA, comme la chlorpromazine, diminue l'activité locomotrice du rat. SEA a un effet inverse de l'adrafinil.

Les valeurs expriment l'activité locomotrice du rat enregistrée pendant 15 minutes après l'administration de SEA, de la chlorpromazine et de l'adrafinil (Moyenne $\pm \mathrm{ESM}, * * * \mathrm{p}<0,001, \mathrm{n}=6$ ).

réflexe de rétablissement chez ces animaux. En présence de la chlorpromazine $(2,5$ $\mathrm{mg} / \mathrm{kg}$ ), le rat n'arrive même pas à s'accrocher à la barre de fer. Par contre, l'injection de l'adrafinil $(10 \mathrm{mg} / \mathrm{kg})$ aux rats provoque une diminution du temps de latence du réflexe de rétablissement qui passe de 2,17 $\pm 0,31$ secondes à $1,17 \pm 0,17$ secondes, soit une réduction de $92,17 \%(P<0,0169)$ par rapport au témoin (Tableau 3). 
Tableau 3: Variation du temps de la latence du réflexe de rétablissement avant et après administration au rat de SEA, de la chlorpromazine et de l'adrafinil.

\begin{tabular}{|c|c|c|}
\hline Substances & Témoin & Test \\
\hline SEA $(10 \mathrm{mg} / \mathrm{kg}$ de PC $)$ & $1,33 \pm 0,21$ secondes & $3,17 \pm 0,48 * *$ secondes \\
\hline $\begin{array}{c}\text { Chlorpromazine } \\
(2,5 \mathrm{mg} / \mathrm{kg} \mathrm{de} \mathrm{PC})\end{array}$ & $1,67 \pm 0,33$ secondes & - \\
\hline Adrafinil $(10 \mathrm{mg} / \mathrm{kg}$ de PC $)$ & $2,17 \pm 0,31$ secondes & $1,17 \pm 0,17$ *secondes \\
\hline
\end{tabular}

SEA augmente le temps de latence du réflexe de rétablissement du rat. SEA a un effet contraire à celui de l'adrafinil. Les valeurs expriment le temps de latence du réflexe de rétablissement du rat avant et après administration de SEA, de la chlorpromazine et de l'adrafinil (Moyenne \pm ESM, *p $<0,05$, **p $<0,01, \mathrm{n}=6$ ).

\section{DISCUSSION}

Les résultats obtenus montrent que l'administration de SEA (10 mg/kg de PC) au rat, provoque une réduction dose-dépendante des activités locomotrice et spontanée et une augmentation de la latence du réflexe de rétablissement. Ces effets sont comparables aux effets de la chlorpromazine $(2,5 \mathrm{mg} / \mathrm{kg}$ de PC), un antipsychotique. Ces effets de SEA, contraires à ceux de l'adrafinil $(10 \mathrm{mg} / \mathrm{kg}$ de PC), un psychostimulant sont toutefois moins importants que ceux de la chlorpromazine, une molécule de référence purifiée en comparaison de l'extrait brut étudié.

Cette diminution de l'activité par SEA, pourrait entrâner une dépression de la fonction mentale (Fritze, 1993 ; Steinberg et al., 1997).

Ces résultats sont comparables à ceux obtenus par Traoré et al. (2003) avec les extraits d'Erythrina senegalensis et d'Heliotropium indicum. En effet, ces auteurs ont montré que l'extrait aqueux de chacune de ces plantes diminuait l'activité spontanée et la locomotion chez le rat.

La diminution de l'activité par SEA, pourrait être aussi due à un effet sédatif de cet extrait aqueux sur le système nerveux. $\mathrm{Ce}$ résultat est similaire à ceux obtenus avec les espèces de Nepeta (Aydin et al., 1998; Rabbani et al., 2008), Salvia reuterana (Rabbani et al., 2005), Stachys lavandulifolia (Rabbani et al., 2003), Echium amoenum (Rabbani et al., 2004) et Valeriana offininalis (Santos et al., 1994).

Elisabetsky et Costa-Campos (2006) ont démontré que les alcaloïdes présents dans les extraits de plantes, seraient à l'origine de leur effet sédatif. Il est donc probable que l'effet de SEA soit lié à la présence de ce composé dans cet extrait aqueux (Néné bi et al., 2009).

SEA induit une réduction dosedépendante de l'activité locomotrice et spontanée du rat et une augmentation de la latence du réflexe de rétablissement. Cet extrait aqueux à un effet sédatif qui serait lié à la présence d'alcaloïde.

\section{REFERENCES BIBLIOGRAPHIQUES}

Adjanohoun E, Adjakidje V, Ahyi MRA, Aké-Assi L, Akoegninou A, D'Almeida J, Apovo F, Boukef K, Chadare M, Cusset G, Dramane K, Eyme J, Gassjta JN, Gbaguidin, Goudotee, Guinko S, Houngnonp, Issalo Kéita A, Kiniffo HV, 
Koné Bamba D, Musampa Nseyya A, Saadou M, Sodogandji T, DE Souza S, Tchabi A, Zinsou Dossa C, Zohoun T. 1989. Contribution aux Etudes Ethnobotaniques et Floristiques au Bénin. Agence de Coopération Culturelle et Technique (ACCT): Paris.

Adjanohoun E, Aké-Assi L. 1979. Contribution au recensement des plantes médicinales de Côte d'Ivoire, Centre national de floristique, Université d'Abidjan.

Aké-Assi L, Guinko S. 1991. Plantes utilisées dans la médecine traditionnelle en Afrique de l'Ouest. Editions Roche Basel, Switzerland.

Ambe GA, Malaisse F. 2000. Les plantes utilisées dans la médicine et la pharmacopée traditionnelles d'une population Malinké en Côte d'Ivoire. Rev.Méd.Pharm.Afr., 14: 121-130.

Aydin S, Beis R, Ozturk Y, Baser KH. 1998. Nepetalactone: a new opioid analgesic from Nepeta caesarea Boiss. J Pharm Pharmacol, 50:813-817.

Bitsindou M, Lejoly J. 1996. Les plantes diarrhéiques chez les Sundi de la souspréfecture de Kindamba (Congo). Academic Publishers.

Dembélé I. 1988. Le tradipraticien et les soins de santé primaires. Thèse de Diplôme d'Etat de Docteur en Médecine, Université d'Abidjan, Abidjan.

Diafouka AJP. 1997. Analyse des usages des plantes médicinales dans 4 régions de Congo-Brazzaville. Thèse de Doctorat, Université libre de Bruxelles, Bruxelles, 431 pages.

Elisabetsky E, Costa-Campos L. 2006. The alkaloid alstonine: a review of its pharmacological properties. $e C A M, \mathbf{3}$ : 39-48.

Fritze J. 1993. Adrenergic-cholinergic imbalance hypothesis of depression: a review and perspective. Rev. Neurosci., 4(1): 63-93.

Néné bi SA, Traoré F, Soro TY, Souza A. 2009. Etude phytochimique et pharmacologique Bridelia ferruginea benth (euphorbiaceae) sur la motricité du Taenia coli de cobaye. Afrique Science, 5(2): 305-320.

Néné bi SA, Traoré F, Zahoui OS, Soro TY. 2008. Composition chimique d'un extrait aqueux de Bridelia ferruginea benth (euphorbiaceae) et études de ses effets toxicologique et pharmacologique chez les mammifères. Afrique Science, 4(2): 287-305.

Olajide OA. 1999. Investigation of the effects of selected medicinal plants on experimental thrombosis. Phytother Res., 13(3): 231-232.

Olajide OA, Makinde JM, Awe SO. 1999. Effects of the aqueous extract of Bridelia ferruginea stem bark on carrageenaninduced oedema and granuloma tissue formation in rats and mice. $J$. Ethnopharmacol., 66(1): 113-117.

Olajide OA, Makinde JM, Okpako DT, Awe SO. 2000. Studies on the antiinflammatory and related pharmacological properties of the aqueous extract of Bridelia ferruginea stem bark. J. Ethnopharmacol., 71(1-2): 153-160.

Olajide OA, Okpako DT, Makinde JM. 2003. Anti-inflammatory properties of Bridelia ferruginea stem bark. Inhibition of lipopolysaccaride-induced septic shock and vascular permeability. $J$. Ethnopharmacol., 88(2-3): 221-224.

Rabbani M, Sajjadi SE, Mohammadi A. 2008. Evaluation of the anxiolytic effect of Nepeta persica Boiss. in mice. eCAM, 5(2): 181-186.

Rabbani M, Sajjadi SE, Zarei HR. 2003. Anxiolytic effects of Stachys lavandulifolia Vahl on the elevated plus- 
maze model of anxiety in mice. $J$ Ethnopharmacol, 89:271-276.

Rabbani M, Sajjadi SE, Jafarian A, Vaseghi G. 2005. Anxiolytic effects of Salvia reuterana Boiss. on the elevated plusmaze model of anxiety in mice. $\mathrm{J}$ Ethnopharmacol, 101:100-103.

Rabbani M, Sajjadi SE, Vaseghi G, Jafarian A. 2004. Anxiolytic effects of Echium amoenum on the elevated plus-maze model of anxiety in mice. Fitoterapia, 75: 457-464.

Santos MS, Ferreira F, Cunha AP, Carvalho AP, Ribeiro CF, Macedo T. 1994. Synaptosomal GABA release as influenced by valerian root extract- involvement of the GABA carrier. Arch. Int. Pharmacodyn Ther., 327: 220-231.

Steinberg BJ, Trestman R, Mitropoulou V, Seby M, Silverman J, Coccaro E, Weston S, DE Vegvar M, Siever LJ. 1997. Depressive response to physostigmine challenge in borderline personality disorder patients. Neuropsychopharmacology, 17(4): 264-273.

Traoré F, Bâ A, Zahoui OS, Néné bi SA. 2003. Effets de Erythrina senegalensis (Fabaceae) et de Heliotropium indicum (Boraginaceae) sur l'activité spontanée et la motricité du Rat. Rev. Iv. Sci. Tech., 4: 83-93. 